\title{
Three-Dimensional Rock Microstructure Modeling Using Two-Dimensional SEM Micrographs
}

\author{
Cheney C.Y. Zhang ${ }^{1,2}$ \\ ${ }^{1}$ Winchester High School, Winchester, MA, USA \\ ${ }^{2}$ DigiM Solution LLC, Burlington, MA, USA
}

As the size of products decreases, microstructures become increasingly important. Examples include fuel cell cathode where micro-porosity increases surface area for energy storage; controlled release drug where interconnected porosity influences release rate and duration; and tight hydrocarbon reservoir where oil and gas in submicron pores dictates hydrocarbon recovery potential. High resolution microscopy imaging is often a must-have tool in characterizing these microstructures. Three-dimensional imaging (3D), such as X-Ray Micro-Computed Tomography (MicroCT), Focused Ion Beam Scanning Electron Microscopy (FIB-SEM), and transmission Electron Tomography (eTomo), are powerful. However, 3D imaging experiment are costly, which limit the potential for broader adoption. Long imaging time and strict sample requirements further refrains the tools from applications involving liquid content and dynamic conditions such as gradients of pressure, temperature, and mass transport. Two-dimensional (2D) micro-images, in comparison, are more easily and plentily available. They provided important insight to the morphology and distributions of the micro-structures, with low cost to information ratio. Quicker imaging time also allows snapshot on transient phenomenon with liquid content.

This project uses 2D SEM micrographs of rock samples to reconstruct 3D micro-structures with two numerical methods. Texture synthesis method [1] is useful to build homogeneous, representative microstructure micrograph from a convoluted micrograph, such as Figure 1a. Due to the large mineral grains and strong charging artifacts, Figure 1a is not suitable for further petrophysical analysis. A small area of intergranular porosity, as shown in Figure $1 \mathrm{~b}$, is the most interested feature, however, it is too small to be representative. Using texture synthesis, a larger, more representative area is generated, Figure 1c. Computing performance is investigated, Figure 1d. which reveals that expanding the microstructure reconstruction to 3D is very time consuming, particularly with the most preferred window size of 13-17. A phase field function method [2] is subsequentially used for full 3D reconstruction. A 2D micrograph is first segmented with an artificial-intelligence based image processing software [3]. The segmentation is then used as boundary condition of phase field function, to reconstruct a corresponding 3D volume. 3D volume initialized with random function appears to provide faster convergence, and higher degree of smoothness of the intergranular porosity network, Figure 2, left panel. In comparison, 3D volume initialized with repetitive 2D boundary image better retains the geometrical irregularity, at the cost of longer computational time.

Ongoing work includes 3D texture synthesis model upon performance improvement, and 3D microstructure evaluation criterion.

References:

[1] A. A. Efros and T. K. Leung. Texture Synthesis by Non-parametric Sampling IEEE International Conference on Computer Vision (ICCV'99), Corfu, Greece, September 1999.

[2] L. Q. Chen. Phase-field models for microstructure evolution. Annual Review of Materials Research, 32:113140,2002

[3] S. Zhang, A. P. Byrnes, J. Jankovic, \& J. Neilly. Management, Analysis and Simulation of Micrographs with Cloud Computing. Microscopy Today 27:1, March 2019. doi:10.1017/S1551929519000026 
Figure 1. Reconstruction of tight sandstone intergranular porosity using GPU accelerated texture synthesis. (a). Original SEM image with heterogeneity and imaging artifact that made it un-suitable for petrophysical modeling; (b). Small area representative for inter-granular porosity; (c). Reconstructed intergranular porosity on a more representatively larger area $(500 \times 500)$ suitable for petrophysical characterization. (d). Computing time with regard to texture kernel size on a 200x200 image.

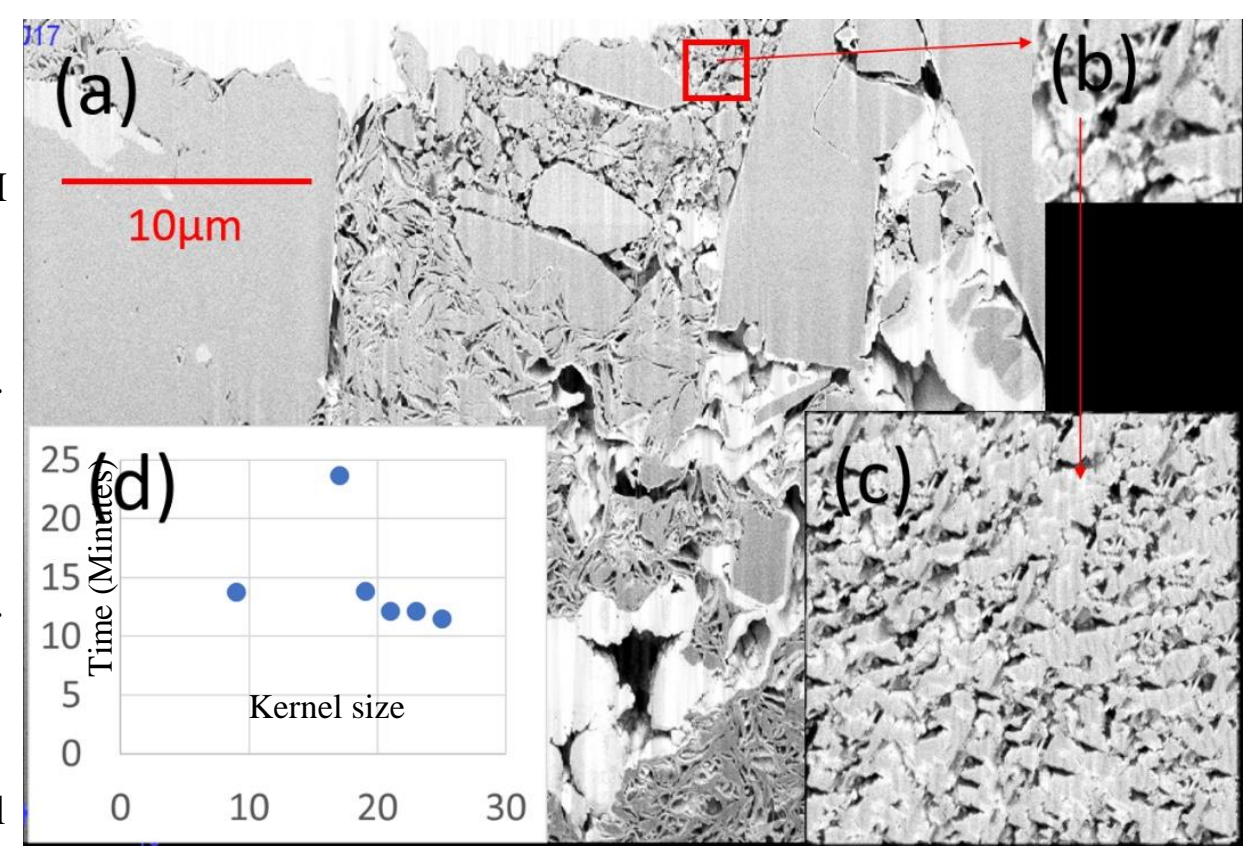

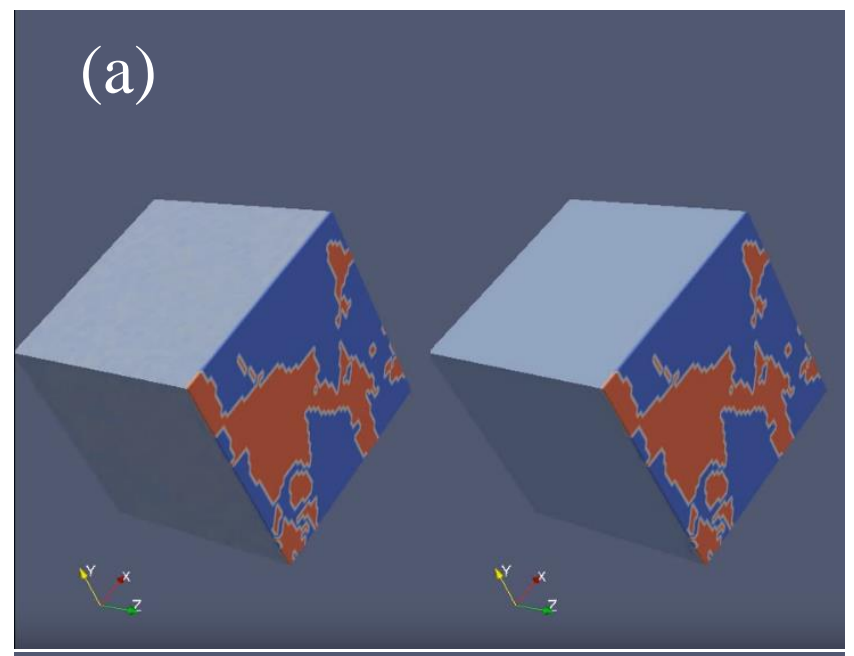

(c)

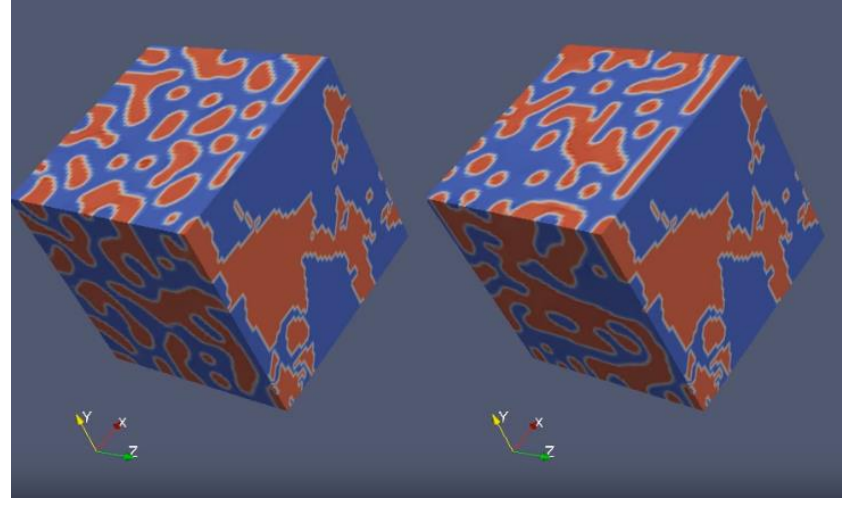

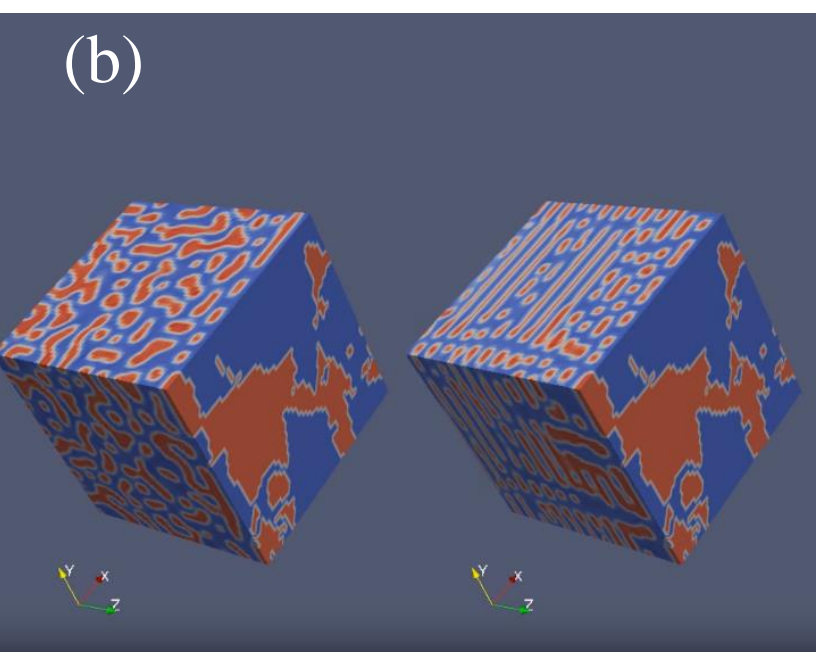

Figure 2. Reconstruction of 3D tight carbonate rock micro-porosity network using phase field simulation initialized with one 2D segmented SEM image. Figures a-c represent steps at increasing iteration numbers. For each figure, the left visualization is from a simulation uses a random function to initialize the 3D domain; the right visualization is from a simulation uses the $2 \mathrm{D}$ boundary image to initialize the full 3D domain. 